\title{
Indicadores de comorbidade em pacientes com Transtorno de Pânico avaliados pelo Método de Rorschach
}

\author{
Paulo Francisco de Castro \\ Universidade de Taubaté - Taubaté, SP, Brasil \\ Universidade Guarulhos - Guarulhos, SP, Brasil
}

\begin{abstract}
Resumo
O presente artigo objetiva a exposição de indicadores de psicopatologia observados em uma amostra de pacientes com Transtorno de Pânico, a partir dos dados do Método de Rorschach. Em síntese, o Transtorno de Pânico pode ser caracterizado como um quadro de vivências extremas de ansiedade, sem aparente motivo, que ocorrem de forma repetida e levam a uma situação paralisadora e à sensação de morte; não raro, observa-se comorbidade do pânico com outros quadros de sofrimento psíquico. O material interpretativo do Método de Rorschach, segundo a proposta do sistema compreensivo, apresenta seis constelações que indicam elementos de cunho psicopatológico ou de sofrimento psíquico. Participaram da investigação 60 sujeitos de ambos os sexos, divididos igualmente em um grupo de pacientes e um grupo de não pacientes. Os resultados indicaram diferenças estatisticamente significativas em duas constelações: maior incidência de PTI $(p=0,002)$ nos pacientes do sexo masculino, revelando que os homens com Transtorno de Pânico tendem a apresentar graves transtornos de percepção e de pensamento; presença de DEPI $(p=0,009)$ onde pacientes com pânico revelam sinais de depressão, sintomas depressivos ou algum tipo de transtorno relacionado à alteração de humor. As demais constelações não diferenciaram os grupos de pacientes e de não pacientes, seus resultados foram CDI ( $\mathrm{p}=0.891)$, S-Con $(\mathrm{p}=1,0)$, HVI $(\mathrm{p}=1,0)$ e OBS $(\mathrm{p}=1,0)$. Assim, na amostra que participou da presente pesquisa, observou-se comorbidade de sintomas depressivos ou de humor na maior parte de todos os pacientes e perturbações severas de pensamento nos pacientes do sexo masculino.
\end{abstract}

Palavras-chave: Avaliação psicológica, Teste de Rorschach, Transtorno de Pânico, Psicopatologia.

\section{Comorbidity indicators in patients with Panic Disorder assessed by the Rorschach Method}

\begin{abstract}
This article aims to show psychopathology indicators obtained in a sample of patients with Panic Disorder from the Rorschach Method. In short, Panic Disorder can be characterized as a condition of extreme anxiety, without an apparent reason, experienced repeatedly and that can lead to a physical or mental paralysis and the sense of impending doom or death; it is not unusual the occurrence of panic comorbidity along with other conditions of psychic suffering. The Comprehensive System for the Rorschach presents six constellations that show elements of psychopathological nature or psychic suffering. Sixty subjects of both sexes participated in the investigation, equally divided into a group of patients and a group of non-patients. Results showed statistically significant differences in two constellations: higher incidence of PTI $(\mathrm{p}=0.002)$ in male patients, showing that men with Panic
\end{abstract}

Endereço para correspondência: Paulo Francisco de Castro. Universidade de Taubaté, Departamento de Psicologia. Avenida Tiradentes, 500, Bom Conselho. Taubaté/SP, Brasil. CEP: 12030-180. Telefone: +55 12 3625 4283. E-mail: pcastro@prof.ung.br.

Nota: Artigo trata de parte ligeiramente modificada da Tese de Doutorado "Caracterização da personalidade de pacientes com Transtorno de Pânico por meio do Método de Rorschach: contribuições do sistema compreensivo", desenvolvida pelo autor junto ao Programa de Pós-graduação em Psicologia Escolar e do Desenvolvimento Humano, no Instituto de Psicologia da Universidade de São Paulo, sob orientação da Professora Doutora Eda Marconi Custódio.

Trabalho apresentado na $41^{\mathrm{a}}$ Reunião Anual da Sociedade Brasileira de Psicologia, na Sessão Coordenada: Avaliação psicológica em processos psicopatológicos. Belém - PA, Outubro de 2011. 
Disorder tend to present serious perception and thought disorders; occurrence of DEPI $(\mathrm{p}=0.009)$ where patients with panic show signs of depression or some kind of depressive symptoms and disorder related to change in humor. The other constellations did not differ between groups of patients and non-patients; their results were CDI ( $\mathrm{p}=0.891)$, S-Con ( $\mathrm{p}=1.0)$, HVI $(\mathrm{p}=1.0)$ and OBS ( $\mathrm{p}=1.0)$. Therefore, in the sample that participated in this research, comorbidity of depressive symptoms or humor was observed in most male patients with severe disturbances of thought.

Keywords: Psychological assessment, Rorschach Test, Panic Disorder, Psychopathology.

\section{Indicadores de comorbilidad en pacientes con Trastorno de Pánico evaluados por el Método de Rorschach}

\section{Resumen}

Este artículo tiene el objetivo de exponer indicadores de psicopatología observados en una muestra de pacientes con Trastorno de Pánico a partir de los datos del Método de Rorschach. En síntesis, el Trastorno de Pánico se puede caracterizar como un cuadro de vivencias extremas de ansiedad, sin motivo aparente, que ocurren repetidamente y llevan a una situación paralizadora y a la sensación de muerte; no es raro observarse co-morbilidad entre el pánico y otros cuadros de sufrimiento psíquico. El material interpretativo del Rorschach, según la propuesta del sistema comprehensivo, presenta seis constelaciones que indican elementos de tipo psicopatológico o de sufrimiento psíquico. Participaron de la investigación 60 sujetos de ambos sexos, divididos igualmente en un grupo de pacientes y un grupo de no pacientes. Los resultados indicaron diferencias estadísticamente significativas en dos constelaciones: mayor incidencia de PTI $(\mathrm{p}=0,002)$ en los pacientes del sexo masculino, revelando que los hombres con Trastorno de Pánico tienden a presentar graves trastornos de percepción y de pensamiento; presencia de DEPI $(\mathrm{p}=0,009)$ donde pacientes con pánico muestran señales de depresión, síntomas depresivos o algún tipo de trastorno relacionado a alteraciones del humor. Las demás constelaciones no mostraron diferencias entre los grupos de pacientes y de no pacientes, sus resultados fueron CDI ( $\mathrm{p}=0.891)$, $\mathrm{S}$-Con $(\mathrm{p}=1,0)$, HVI $(\mathrm{p}=1,0)$ y OBS $(\mathrm{p}=1,0)$. Así, en la muestra que participó de esta investigación se observó co-morbilidad entre síntomas depresivos o del humor en la mayor parte de los pacientes con perturbaciones severas del pensamiento en los pacientes del sexo masculino.

Palabras-clave: Evaluación psicológica, Test de Rorschach, Trastorno de Pánico, Psicopatología.

\section{Introdução}

\section{Breve introdução sobre o Transtorno de Pânico}

Muito se discute a respeito dos transtornos de pânico, seus sintomas e diagnóstico, mas, em certos casos, é de opinião comum que existe dificuldade para se chegar a uma conclusão precisa, em razão da grande variedade dos sintomas e de sua intensidade. O Método de Rorschach pode ser importante estratégia para investigação psicodiagnóstica nesses casos.

Quando se trata de transtorno de pânico, não se pode deixar de citar certa dificuldade diagnóstica e, nos relatos de casos (Gentil, 1997a), pode-se verificar que se trata de um quadro em que profissionais tendem a cometer enganos diagnósticos, quer por desconhecimento do conjunto de sintomas, uma vez que o desencadeamento de ataques de pânico envolve mecanismos patológicos complexos, integrando diferentes estruturas biológicas e funcionamentos cognitivos (Ramos, 1995), quer por desconsiderar a gravidade do quadro.

A angústia e o estresse desencadeados pela dificuldade em se estabelecer rapidamente um diagnóstico agravam o quadro de pânico, fazendo com que este se intensifique e, consequentemente, dificulte seu tratamento (Troiano, 2001).

Em termos genéricos, o transtorno de pânico pode ser caracterizado pela incidência recorrente de ataques de pânico, em razão de uma crise aguda de ansiedade, cujo indivíduo vive um mal-estar e sensação iminente de 
perigo e/ou morte (Gentil, 1997b; Vieira, 1997). Embora possa ser classificada como um afeto positivo, quando a ansiedade torna-se excessiva ou geradora de sofrimento psicológico esta passa a ser considerada patológica (Cheniaux, 2011; Dalgalarrondo, 2008).

Os manuais descritivos apresentam a definição de Transtorno de Pânico com sendo a presença de ataques de ansiedade grave e pânico que ocorrem inesperadamente e sem qualquer situação desencadeante, que se configuram recorrentes, acompanhados de, pelo menos um período de um mês, pela preocupação constante de que ocorra outra crise e suas implicações, associado a mudanças comportamentais decorrentes da vivência da crise de pânico. Embora os sintomas variem para cada indivíduo, observa-se palpitações, dor no peito, sensações de choque, tontura, e sentimentos de irrealidade (despersonalização ou desrealização), observa-se ainda grande temor por morrer, perder o controle ou ficar louco. Importante ressaltar que as crises de pânico não são decorrentes de efeitos fisiopatológicos ou de condições médicas (American Psychiatric Association [APA], 1995; Organização Mundial da Saúde [OMS], 1992/1993).

Para Arbona e Arnal (1995), o transtorno de pânico constitui-se, a partir da década de 1980, como uma importante patologia para investigações por se tratar de um grande problema de saúde pública nas sociedades industrializadas. Os autores esclarecem que, em razão de sua incidência, várias pesquisas preocuparam-se em refletir sobre a etiologia e o tratamento mais adequado nestes quadros, questionando modelos teóricos provenientes tanto da Psiquiatria como da Psicologia, assim como dos estudos sobre ansiedade e seus distúrbios.

Segundo Del-Ben (2004), as primeiras descrições nosológicas do que é denominado atualmente de Transtorno de Pânico datam do século XIX, onde soldados que atuaram na guerra civil americana apresentaram sintomatologia física (palpitações, dores torácicas e outros sintomas cardíacos) sem a presença de lesões orgânicas. Em outro momento histórico, muitos autores associam o conceito freudiano de neurose de angústia com a compreensão que se tem sobre Transtorno de Pânico (Arbona \& Arnal, 1995; Del-Ben, 2004). Depois disso, observou-se que o termo "pânico" foi introduzido, pela primeira vez, em
1962 por Klein e Fink $^{1}$, quando os autores substituíram a terminologia ataques de ansiedade por ataques de pânico, reforçando as diferenças entre as crises de ansiedade e a vivência de ansiedade do tipo antecipatória no que se refere à sua etiopatogenia (Arbona \& Arnal, 1995).

Para Gentil (1996), um ataque de pânico que, geralmente, é inesperado e intenso, pode ser uma experiência avassaladora. Os sintomas vivenciados pelo paciente e as sensações de fadiga e desconforto sentidas após a crise são extremamente desagradáveis e levam a uma inquietação e temor de outra ocorrência.

Na descrição bibliográfica pesquisada, há uma grande convergência na caracterização da crise de pânico pelos diferentes autores, cujo termo é entendido como um período de intenso medo, apreensão e perigo, gerado de forma espontânea, frequentemente, acompanhado de sensação iminente de enlouquecimento, perigo ou morte. Após a crise, o paciente experimenta um grande desejo de sair do ambiente onde a mesma ocorreu (Arbona \& Arnal, 1995; DelBen, 2004; Gentil, 1996; Gentil, 1997b; Kaplan \& Sadock, 1991/1993; López, 1995).

De qualquer forma, apesar disso, "os ataques de pânico são experiências ambíguas, abertas a interpretações múltiplas e mutáveis" (López, 1995, p. 30), em cuja periodicidade pode demonstrar tanto a gravidade do caso como a intensidade do distúrbio (López, 2000).

\section{O Método de Rorschach na Investigação de Aspectos Psicopatológicos}

O Método de Rorschach organizou-se e configurou-se como uma das mais importantes técnicas de investigação psicológica, pautandose em sua base fundamentalmente clínica. Oriundo do desenvolvimento da psiquiatria e da psicanálise na época de sua criação, despertou grande interesse científico na área, conquistando ampla confiabilidade entre os profissionais de saúde mental no período (Vaz, 1997; Yazigi, 2010).

Assim, o Rorschach é, tradicionalmente, classificado como um instrumento para avaliação de condições normais ou patológicas dos indivíduos (Weiner, 1986). É constituído

\footnotetext{
${ }^{1}$ Referência original: Klein, D. F.; Flink, M. (1962). Psychiatric reaction patterns to imipramine. American Journal of Psychiatry, 2, 14-17.
} 
com base nas comparações entre pacientes que apresentavam um sofrimento mental e pessoas da população comum; pode-se dizer que o método proposto por Rorschach nasceu fundamentado em informações obtidas em funcionamentos psicopatológicos, por isso, talvez, nenhuma outra técnica de avaliação psicológica possa, com tanta precisão, avaliar a psicopatologia de pacientes (Yazigi, 2010).

É importante ressaltar o grande número de pesquisas que foram - e ainda são desenvolvidas na área de psicopatologia com o método. Desde sua publicação, um grande número de clínicos e pesquisadores desenvolveu trabalhos com o Rorschach e as mais diferentes formas de psicopatologias.

O conhecimento acumulado sobre a aplicação do Rorschach nas mais variadas psicopatologias permite uma constante revisão dos conceitos de seus diagnósticos diferenciais, acompanhando a evolução dos quadros e as descobertas científicas da área. Todo clínico ou pesquisador que utiliza o Rorschach para investigar a psicopatologia espera que seus estudos auxiliem nas próximas investigações e esperam incentivar os jovens clínicos e pesquisadores a usar também o Método de Rorschach, mantendo aceso o interesse e o desenvolvimento do instrumento (Campo, 1995; Chabert, 1987/1993; Cunha, 1996; Nascimento, 2010; Santos, 1996; Vaz, 1997). Com este instrumento, é possível tipificar e diferenciar quadros diagnósticos, levando o psicólogo a uma conclusão mais fiel e a um planejamento terapêutico mais direcionado ou o pesquisador a delineamentos mais precisos e a considerações mais claras de seu trabalho.

Segundo Frank (1997), a possibilidade do uso clínico do Rorschach como instrumento no psicodiagnóstico diferencial transcende seus dados psicométricos. Os aspectos quantitativos constituem um importante referencial quando se precisa realizar um diagnóstico que tem como objetivo avaliar as diferenças significativas entre um funcionamento típico e um patológico. Além disso, a possibilidade de uma leitura simbólica das verbalizações possibilita uma compreensão psicodinâmica bastante específica, necessária para a contextualização individual do paciente que está sendo avaliado.

Para que os dados quantitativos do Rorschach em um contexto clínico sejam usados na realização do psicodiagnóstico diferencial, são necessárias investigações anteriores que fundamentem os dados para uma comparação precisa, sobretudo, no que se refere à avaliação de manifestações psicopatológicas. Necessita-se de pesquisas específicas, cujo objeto de estudo seja as mais diferentes patologias e, com base em investigações empíricas, levantar os dados e os índices específicos, para que as futuras avaliações apoiem-se em proposições testadas e comprovadas e não em especulações sem sua devida constatação (Weiner, 1986).

Em específico no que tange ao transtorno de pânico, pelos trabalhos realizados por Andersen e Rosenberg (1990) e Adrados e Figueiredo (1995), percebe-se que o Rorschach é um instrumento capaz de caracterizar com precisão a síndrome e suas motivações psíquicas, tanto estruturais como inconscientes.

\section{Estudo do pânico por meio do Método de Rorschach}

Baseado no Sistema Compreensivo, o Rorschach foi objeto de pesquisa de Andersen e Rosenberg (1990) no estudo comparativo entre 41 pacientes com transtorno de pânico, 14 com depressão maior e 18 com desordens de ansiedade generalizada, cujos dados foram comparados com 18 sujeitos normais.

Após a comparação dos dados, nos protocolos dos pacientes com Transtorno de Pânico foram observadas diferenças significativas nos seguintes itens: diminuição do número de respostas dos testes - $\mathrm{R}(\mathrm{p}<$ $0,01)$ revelando rebaixamento da produtividade; diminuição das respostas com determinante de movimento humano - $\mathrm{M}$ ( $\mathrm{p}<$ $0,05)$ que indica dificuldade de elaboração interna mais consistente; diminuição das respostas de cor - WsumC ( $\mathrm{p}<0,05)$ que expõe a dificuldade dos pacientes em lidar com afetos e sentimentos; diminuição da porcentagem de respostas com localização de detalhe comum $\mathrm{D}(\mathrm{p}<0,05)$ que trata da dificuldade de solucionar problemas de forma mais prática, considerando as condições da realidade; rebaixamento da porcentagem das respostas de forma bem organizadas e integradas $-\mathrm{FQ}+\mathrm{e}$ FQo ( $\mathrm{p}<0,05$ ), indicando um afastamento do pensamento mais convencional, que considera a realidade objetiva; aumento da porcentagem de respostas de conteúdo humano - $\mathrm{H}(\mathrm{p}<0,01)$ que é interpretado como uma maior preocupação na percepção de si e dos demais seres humanos; diminuição da atividade 
organizativa das respostas - Zf $(\mathrm{p}<0,01)$ que revela pouca capacidade criativa e diminuição do esforço em criar.

Por fim, os autores observaram uma diminuição das respostas com determinantes mistos - Blends ( $\mathrm{p}<0,001)$, indicando pobreza associativa (Andersen \& Rosenberg,1990). Os autores concluíram o trabalho, expondo que os pacientes com transtorno de pânico indicaram um comprometimento na estrutura da personalidade e implicações na habilidade dos processos cognitivos. Como os resultados mostraram-se muito relevantes no estudo do pânico, salientaram que o emprego do Rorschach é de extrema valia no diagnóstico diferencial das diferentes psicopatologias, inclusive do Transtorno de Pânico (Andersen \& Rosenberg, 1990).

Cohen e Ruiter (1992) testaram 22 pacientes, com idade entre 24 e 50 anos, com transtorno de pânico associado à agorafobia, empregando o Rorschach segundo o sistema compreensivo. Compararam seus resultados com os dados de outros quadros clínicos publicados (esquizofrenia, distúrbios de sono e depressão), além de dados publicados de não pacientes. Os dados da pesquisa revelaram um rebaixamento do índice de reação afetiva (Afr) e um rebaixamento do valor ponderado das respostas de cor cromática (WsumC) dos pacientes de uma forma geral, quando comparados aos indivíduos não pacientes. Tal aspecto revelou características de fuga diante do contato afetivo, gerando certa restrição na vida afetiva dos pacientes, fazendo com que estes usassem um mecanismo de formação reativa para se defender da necessidade de afeto que sentiam.

No ano seguinte, os mesmos autores desenvolveram uma investigação com 18 pacientes holandeses com pânico associado à agorafobia e quatro pacientes holandeses com pânico sem agorafobia. Usaram também o Rorschach baseado no sistema compreensivo, identificando uma dificuldade na habilidade dos pacientes em lidar com emoções negativas e ansiogênicas, associadas a uma falha dos processos defensivos (Cohen \& Ruiter, 1993).

MacFadden (1994) estudou nove pacientes do sexo feminino com diagnóstico de pânico, por meio do Método de Rorschach, segundo o sistema de classificação de Silveira. Após a análise das médias obtidas nos protocolos das pacientes, comparou os resultados aos valores normativos propostos pelo sistema, obtendo-se as seguintes diferenças: aumento da porcentagem do número de respostas $(\% \mathrm{R}$ média 69,82), revelando uma adequação do rendimento do trabalho mental desses sujeitos; diminuição da porcentagem de respostas determinadas pela forma de boa qualidade $(\% \mathrm{~F}+$ - média 63,50$)$ que significa uma baixa susceptibilidade aos diferentes estímulos do ambiente; aumento da porcentagem de respostas com conteúdo animal $(\% \mathrm{~A}$ - média 45,82 ) que demonstra dificuldade em estabelecer relacionamentos interpessoais de forma adulta; diminuição do coeficiente de elaboração intelectual das respostas (elab/R média 0,89 ), indicando que a produção mental das pacientes foi pobre, restrita e pouco criativa; aumento do índice afetivo (Af - média $1,49)$, apresentando grande sensibilidade às estimulações afetivas advindas do meio, sobretudo as mais básicas e primárias; aumento do índice de impulsividade (Imp - média 0,71) que significa falta de controle de sentimentos mais primitivos; diminuição do índice de Conação (Con - média 33,3) que mostra uma dificuldade subjetiva para agir no mundo exterior; diminuição do índice Lambda (L média 0,43 ) oferece dados que permitem dizer que há restrição dos recursos íntimos da personalidade para a elaboração de seus conteúdos (MacFadden, 1994).

Outro estudo empregando o Método de Rorschach usou o sistema de classificação de Klopfer, trata-se do estudo desenvolvido por Adrados e Figueiredo (1995) a partir de um levantamento de 85 protocolos de pacientes diagnosticados clinicamente como portadores de Síndrome do Pânico, de ambos os sexos. Para as autoras, a maioria dos pacientes que participou do estudo percebeu os estímulos do meio externo de forma equilibrada e satisfatória (porcentagem de respostas de forma - F\% adequada); houve um predomínio de uma posição vivencial extratensiva (predomínio das respostas de cor sobre as de movimento); manutenção da capacidade de crítica diante dos acontecimentos rotineiros ( $\mathrm{F}+$ extenso dentro dos padrões para a população).

As autoras justificam esse dado, entendendo que, após as crises, os pacientes conseguem reorganizar sua estrutura egoica. Elas indicaram certa imaturidade no grupo do sexo feminino (respostas de movimento animal - FM - maior do que as respostas de movimento humano - $\mathrm{M})$; excessiva emotividade e labilidade afetiva, levando-os a reações 
emocionais intensas e impulsivas (respostas de cor com características formais secundárias - C $+\mathrm{CF}$ - maior do que a incidência de respostas de forma com cor - FC). Por fim, revelaram que o volume de ansiedade estava aumentado em relação a grupos com as mesmas características culturais, este aspecto foi mais intenso nas mulheres que participaram do estudo (respostas com determinantes de sombreado aumentada, quando comparados aos dados normativos textura - Fc, $\mathrm{cF}$ e c, profundidade - FK, difusão - KF e K) (Adrados \& Figueiredo, 1995).

Em estudo desenvolvido por Sarvasi (1999), foram investigadas as características das relações objetais estabelecidas por cinco pacientes com pânico; para tanto, foi usado o Método de Rorschach sob dois enfoques: a aplicação segundo o modelo proposto por Klopfer e a atualização dos procedimentos baseados no Rorschach Temático. A autora notou que quatro entre os cinco pacientes apresentaram menor incidência de respostas de movimento humano quando comparadas à incidência de respostas dos movimentos animal e inanimado $(M<F M+m)$, revelando maior uso de fantasias em suas elaborações internas, este aspecto também está associado a pouca capacidade de sublimação nesses pacientes.

No estudo dos fenômenos especiais, a autora observou a incidência em quatro sujeitos dos seguintes aspectos: giro instantâneo do cartão, que revela desconfiança e conflito entre autonomia e dependência, mobilizando de forma prematura condutas de oposicionismo e atuação; respostas de par, indicando certo narcisismo e, por fim, choque ao vazio, traduzindo fantasias de abandono da mãe e uma percepção negativa da imagem materna (Sarvasi, 1999).

Pautada em uma leitura das relações objetais, percebeu, a partir da articulação das histórias narradas, que os pacientes apresentaram um funcionamento específico das configurações das relações objetais, como necessidade de apoio da figura materna, gerando uma percepção negativa dessa figura, com base nas vivências de abandono; uso de identificação projetiva como mecanismo de defesa principal; conflito entre autonomia e dependência (Sarvasi, 1999).

Farah e Villemor-Amaral (2008), em pesquisa com pacientes com pânico, utilizando o Rorschach no sistema compreensivo, concluem que esses indivíduos indicam expressões emocionais muito intensas, sugerindo certa impulsividade, além de revelar certa labilidade na interação emocional. Levantaram também sentimentos intensos de vazio emocional associado às vivências destrutivas.

Assim, observa-se relevante utilização do Método de Rorschach no estudo do Transtorno de Pânico. Apesar de vastamente empregado, justifica-se o presente estudo pela possibilidade de discutir elementos relacionados aos indicadores de comorbidade do quadro com outras psicopatologias.

\section{Objetivo}

O objetivo do presente artigo é discutir os indicadores psicopatológicos observados em pacientes com Transtorno de Pânico, avaliados pelo Método de Rorschach.

\section{Método}

A importância de um estudo empírico está em proporcionar a análise de um fenômeno a ser pesquisado de forma coerente e real, considerando-se todos os aspectos apresentados pelos sujeitos sob influência de variáveis ambientais, fundamentadas na intercorrelação que estes fenômenos podem apresentar (Hernández, 1993).

A interpretação dos dados empíricos, baseada no estudo das variáveis que serão indicadas, possibilita uma intensa articulação entre o lógico e o real, ou seja, entre o conhecimento acumulado e descrito nos pressupostos teóricos e a apresentação viva do fenômeno a ser estudado nos resultados apresentados pelos sujeitos, proporcionando uma efetivação e um crescimento do conhecimento científico (Hübner-D’Oliveira, 1984; Severino, 2001).

\section{Participantes}

Participaram da pesquisa um total de 60 pessoas, divididas em dois grupos homogêneos e equivalentes para o estudo da personalidade dos pacientes com pânico: o Grupo I, denominado Grupo de Pacientes e o Grupo II, para efeitos de controle, que foi chamado Grupo de não pacientes.

Para compor o Grupo de Pacientes (Grupo I), fizeram parte da amostra 30 sujeitos, sendo 15 do sexo feminino e 15 do sexo masculino, todos com diagnóstico de transtorno de pânico sem comorbidade, inscritos em programas existentes em um Ambulatório de Saúde 
Mental de uma cidade da Grande São Paulo, para o atendimento dessa patologia.

Para compor o Grupo de Não pacientes (Grupo II) 30 sujeitos foram analisados, sem qualquer tipo de queixa, com idade, nível de escolaridade e nível socioeconômico equivalentes aos obtidos no Grupo I, divididos em 15 do sexo feminino e 15 do sexo masculino. Alguns participantes do Grupo II faziam parte da comunidade acadêmica da Universidade (funcionários e estudantes de outros cursos) que indicaram outros colaboradores para participarem da pesquisa.

Houve um pareamento direto quanto à escolaridade. No que se refere à idade dos sujeitos, a média geral das idades dos pacientes foi de 42,2 anos e dos não pacientes 42,5. Quando o sexo dos colaboradores é considerado, para o sexo feminino tem-se média em 45,2 às pacientes e 45,3 às não pacientes; para o sexo masculino, as médias de idade foram 39,2 aos pacientes e 39,7 aos não pacientes. As médias obtidas a partir da idade dos colaboradores evidenciam semelhança entre os dois grupos.

\section{Instrumentos para a Coleta de Dados}

Foram realizadas entrevistas dirigidas com os participantes. Os roteiros das entrevistas foram desenvolvidos com a finalidade de levantar dados de caracterização da amostra, tanto de pacientes, como de não pacientes. Nesse sentido, foram levantadas informações gerais de cada sujeito como: idade, sexo, escolaridade, profissão, ocupação e estado civil. As questões que compuseram os questionários foram desenvolvidas baseadas no quadro dos sintomas de pânico apresentados pelo DSM-IV (APA, 1995), explicitado e detalhado pelo material bibliográfico estudado, sobretudo de Barlow e Cerny (1988/1999) e López (2000). Estes autores foram escolhidos por tratarem especificadamente de procedimentos psicológicos no diagnóstico do pânico.

Em razão de sua grande eficácia e utilidade para a análise do pânico, optou-se pela estrutura aplicada em alguns inventários que objetivam uma caracterização clara deste quadro (Gentil, Ito, \& Roso 1987; Ito \& Ramos, 1998; López, 2000), pois a proposta foi de que cada um dos sintomas investigados siga um modelo de graduação o que possibilita a investigação não só dos sintomas específicos, mas também de suas intensidades. $\mathrm{O}$ roteiro para os sujeitos não pacientes foi desenvolvido, pautando-se no original para os pacientes, adaptando-se a investigação para indivíduos que não sofreram crises de pânico.

No roteiro do questionário, empregou-se uma estrutura similar a de uma escala, com base em itens distribuídos no tipo Likert pela sua objetividade, o que permite uma padronização a respeito das informações a serem coletadas, facilitando a análise quantitativa dos elementos investigados. Assim, os questionamentos distribuídos em uma escala de investigação diagnóstica podem apresentar um padrão sintomatológico do quadro, possibilitando comparar os resultados ao grupo controle (Ito \& Ramos, 1998).

Para verificação do estado de saúde geral dos participantes do grupo de não pacientes, foi utilizado o Questionário de Saúde Geral de Goldberg (QSG), que é um instrumento de avaliação psicológica, com estrutura de autorrelato, composto por 60 questões fechadas. A tarefa do indivíduo avaliado é responder cada um dos itens em uma folha de respostas, comparando seu estado psicológico usual com uma das alternativas propostas em uma escala do tipo Likert de quatro pontos. Segundo Cunha (2000), o QSG foi delineado para avaliar a gravidade do estado psicopatológico sem características psicóticas em indivíduos para efeito de triagem.

$\mathrm{O}$ referido instrumento avalia cinco aspectos específicos de saúde geral, sendo estresse psíquico, desejo de morte, desconfiança no desempenho, distúrbios do sono e distúrbios psicossomáticos. Quando os escores desses cinco fatores são somados, compõe-se o escore de saúde geral (Goldberg, 1972/1996). Para efeito deste estudo, foi utilizado o escore geral do QSG de cada um dos participantes.

Além dos roteiros de entrevista e QSG, todos os participantes foram submetidos ao Método de Rorschach, de acordo com as especificações técnicas do sistema compreensivo (Exner, 1993/1994, 1995, 1995/1999). No presente trabalho, apenas serão mencionados os resultados do Rorschach.

\section{Procedimento para a Coleta de Dados}

Conseguiu-se a autorização para a coleta de dados em um Ambulatório de Saúde Mental situado na Grande São Paulo, onde foi possível utilizar a estrutura da instituição para o contato com os pacientes, bem como para aplicação do questionário e do Método de Rorschach. 
O prontuário dos pacientes do Ambulatório foi consultado no sentido de separar os que sofriam de Transtorno de Pânico, sem comorbidades, que foram convidados, por telefone ou por correspondência, a participar da pesquisa.

Para os que aceitaram, foi agendado um dia para as atividades. Em um primeiro momento, a Carta de Informação sobre a Pesquisa e o Termo de Consentimento Livre e Esclarecido foi entregue, conforme normas estabelecidas para pesquisas envolvendo seres humanos dispostas pelo Ministério da Saúde (Brasil, 1996).

Após as explicações necessárias e diante do aceite dos colaboradores, foi realizada a entrevista sobre os dados gerais e o levantamento de sintomas, bem como a aplicação do Método de Rorschach, de acordo com a disponibilidade de cada um dos colaboradores. A maior parte dos pacientes agendou as atividades para um dia em que tinha alguma atividade no Ambulatório.

A aplicação do Método de Rorschach seguiu, rigorosamente, todas as especificações técnicas propostas pelo sistema compreensivo (Exner, 1993/1994, 1995, 1995/1999).

A coleta de dados dos colaboradores do Grupo I (pacientes) ocorreu nas dependências do Ambulatório de Saúde Mental, em ambiente adequado às aplicações, segundo as especificações técnicas. A instituição cedeu uma sala para que ocorressem as aplicações. A coleta de dados do sexo feminino terminou antes que todos os dados fossem levantados. Dessa forma, durante um período final de levantamento esperou-se a colaboração apenas de pacientes do sexo masculino.

Após o término da coleta de dados dos pacientes, procedeu-se ao levantamento da idade e escolaridade para o convite aos colaboradores do Grupo II (não pacientes). Os indivíduos que preenchiam os requisitos de idade e escolaridade eram submetidos ao QSG para verificação do estado psicológico geral. Foi divulgado um convite à comunidade acadêmica (funcionários e alunos de graduação - exceto do Curso de Psicologia), sobre a participação da pesquisa, estes poderiam indicar outros indivíduos para a aplicação dos testes.

Para fazer parte da pesquisa, os participantes do Grupo II foram submetidos individualmente ao Questionário de Saúde Geral - QSG (Goldberg, 1972/1996) para verificação das características psicológicas gerais, garantindo-se que estes não possuíam nenhum quadro de sofrimento psicológico, quer leve ou severo. O QSG mostrou-se como uma estratégia eficaz para a seleção dos indivíduos.

Após a seleção da amostra, os participantes eram submetidos ao Método de Rorschach. A aplicação para os colaboradores não pacientes ocorreu na Clínica Psicológica de uma Universidade situada na Grande São Paulo, em ambiente adequado para as atividades.

Todos os participantes assinaram o Termo de Consentimento Livre e Esclarecido, de acordo com as normas de pesquisa com seres humanos. Cabe ressaltar que todos os procedimentos da pesquisa foram avaliados e aprovados pelo Comitê de Ética em Pesquisa com Seres Humanos do Instituto de Psicologia da Universidade de São Paulo, conforme Ofício Of. 1206/CEPH.

\section{Procedimento de Análise dos Dados}

As respostas obtidas nas aplicações do Método de Rorschach foram codificadas, considerando-se todos os aspectos propostos pelo sistema compreensivo (Exner, 1993/1994, 1995/1999).

Toda a codificação das respostas foi realizada por dois juízes, sendo um deles o autor da pesquisa e o outro, professor do Método de Rorschach, membro da Associação Brasileira de Rorschach e Métodos Projetivos e com formação no sistema compreensivo. Ambos codificaram, independentemente, cada protocolo. A análise da incidência dos itens de codificação realizada pelos juízes seguiu à seguinte estratégia:

- Concordância entre os dois juízes - item de codificação foi aceito diretamente para análise;

- Discordância entre os dois juízes - o item foi analisado por um terceiro juiz, igualmente especializado no sistema compreensivo que decidiu entre as duas posições diferentes.

Foram obtidos 60 protocolos, perfazendo um total de 1.133 respostas que foram analisadas pelos dois juízes. Após a comparação da codificação realizada, observou-se que, em 29 protocolos (48,3\%), houve concordância total entre os dois avaliadores e, em 1.072 respostas (94,6\%), também ocorreu uma concordância total nas codificações. Dessa maneira, quando as 
respostas foram consideradas separadamente, observou-se que a discrepância das codificações entre os avaliadores foi mínima.

Dos protocolos analisados, 31 (51,7\%) apresentaram algum tipo de discordância, perfazendo um total geral de 61 respostas $(5,4 \%)$ com algum tipo de diferença entre os dois avaliadores.

No que se refere ao tipo de discordância observada, tem-se que os itens de maior diferença foram relacionados às determinantes e aos códigos especiais (21 diferenças cada um), seguidos da divergência de conteúdos (12 diferenças) e qualidade evolutiva (11 diferenças). Os itens com menor quantidade de divergências foram: a localização (duas diferenças) e respostas em par (uma diferença). Os itens de codificação relacionados à qualidade formal, respostas populares e valor de elaboração não indicaram diferença na codificação entre os dois avaliadores.

As diferenças foram analisadas por um terceiro juiz que optou entre as duas codificações apontadas. Dessa forma, foi possível diminuir a interferência pessoal na codificação das respostas. Após a decisão do terceiro juiz, as respostas foram cotadas para a organização do Sumário Estrutural de cada um dos protocolos.

Os dados foram avaliados de acordo com os itens que compõem as constelações propostas pelo sistema compreensivo, divididos em quatro grupos definidos como: $\mathrm{PF}, \mathrm{PM}, \mathrm{CF}$ e CM para o tratamento estatístico.

Para descrever o perfil da amostra segundo as variáveis em estudo, foram feitas tabelas de frequência das variáveis categóricas (classificação dos parâmetros de personalidade identificados pelo Rorschach), com valores de frequência absoluta $(\mathrm{N})$ e porcentual $(\%)$, e estatísticas descritivas das variáveis contínuas (escores obtidos no Método de Rorschach), com valores de média, desvio-padrão, valores mínimo, máximo e mediana (Levin, 1987; Pereira, 1999; Siegel, 1975).

Para comparação das variáveis categóricas entre os quatro grupos, foi utilizado o teste QuiQuadrado ou, quando necessário, o teste exato de Fisher (presença de valores esperados menores que 5). Para comparar as variáveis numéricas ou contínuas entre os quatro grupos, foi empregado o teste de Kruskal-Wallis (Levin, 1987; Pereira, 1999; Siegel, 1975), em razão do tamanho dos grupos e da ausência de distribuição Normal das variáveis. As comparações múltiplas entre os quatro grupos foram feitas por meio do teste post-hoc de Dunn (Levin, 1987; Pereira, 1999; Siegel, 1975). O nível de significância adotado para os testes estatísticos foi de 5\%, ou seja, $\mathrm{p}<0.05$.

Os aspectos mais significativos que caracterizaram o grupo de pacientes foram identificados. A seguir, foram feitos os agrupamentos dos itens que compõem as constelações para a interpretação proposta pelo sistema compreensivo (Acklin, 1995; Exner, 1993/1994; Exner \& Sendín, 1998/1999; Weiner, 1998/2000).

\section{Resultados e discussão}

Após a codificação das respostas, os dados foram analisados de acordo com sua incidência e tratamento estatístico. A seguir, há a exposição das seis constelações propostas pelo sistema compreensivo, com vistas à verificação de comorbidades presentes nos pacientes com pânico.

São demonimadas de constelações por se tratar de um agrupamento de sinais específicos para cada quadro, que formam um índice de verificação de comprometimento psicológico em aspectos específicos da personalidade.

Nesta discussão, as descrições das constelações e seus respectivos significados interpretativos apresentados foram baseados nos manuais técnicos sobre o Método de Rorschach, segundo o sistema compreensivo (Exner, 1993/1994; Exner, 1995/1999; Exner \& Sendín, 1998/1999; Nascimento, 2010).

\section{Índice de Depressão}

O Índice de Depressão - DEPI é obtido pela observação de 15 variáveis que se articularam em sete elementos de análise. $\mathrm{O}$ índice seria considerado positivo, se houvesse a presença de, pelo menos, cinco desses elementos. Quando positivo, o DEPI indica a possibilidade de uma vivência depressiva ou algum tipo de transtorno afetivo, indicando ainda que a organização psicológica do indivíduo é bastante vulnerável à depressão ou alterações de humor.

No que se refere à comparação dos valores obtidos em DEPI, expostos nos dados das Tabelas 1 e 2, foram verificadas diferenças significativas entre os grupos pesquisados. 
Tabela 1 - Presença de DEPI nos quatro grupos pelo Teste Exato de Fisher

\begin{tabular}{llllll}
\hline DEPI & Sim & \multicolumn{3}{c}{ Não } & Sig \\
\cline { 2 - 5 } & $\mathrm{N}$ & $\%$ & $\mathrm{~N}$ & $\%$ & \\
\hline $\mathrm{PF}$ & 7 & 46,67 & 8 & 53,33 & \multirow{2}{*}{$\mathrm{p}=0,009$} \\
$\mathrm{PM}$ & 8 & 53,33 & 7 & 46.67 & \\
$\mathrm{CF}$ & 2 & 13,33 & 13 & 86,67 & \\
$\mathrm{CM}$ & 1 & 6,67 & 14 & 93,33 & \\
\hline
\end{tabular}

Tabela 2 - Incidência de indicadores de DEPI nos quatro grupos pelo teste de Kruskal-Wallis

\begin{tabular}{ccccccc}
\hline Indic. & Média & D.P. & Min & Máx & Mediana & Sig \\
\hline PF & 4,40 & 0,63 & 3,00 & 5,00 & 4,00 & \\
PM & 4,40 & 0,99 & 2,00 & 6,00 & 5,00 & $p<0,001$ \\
CF & 3,47 & 0,83 & 2,00 & 5,00 & 3,00 & \\
CM & 3,33 & 0,72 & 2,00 & 5,00 & 3,00 & \\
\hline
\end{tabular}

Como é possível verificar nos dados da Tabela 1, os grupos de pacientes apresentaram maior incidência de DEPI positivo, $46,67 \%$ $(\mathrm{N}=7)$ nos pacientes do sexo feminino e 53,33 $(\mathrm{N}=8)$ nos pacientes do sexo masculino. $\mathrm{Na}$ observação dos dados do grupo-controle, esta incidência caiu para $13,33 \%(\mathrm{~N}=2)$ no controle feminino, e $6,67 \%(\mathrm{~N}=1)$ no masculino.

Após comparação das variáveis categóricas do Índice de Depressão pelo teste Exato de Fischer, obteve-se a diferença $\mathrm{p}=$ 0,009 , indicando que houve uma diferença estatisticamente significativa entre os dois grupos, sendo maior a incidência do Índice de Depressão no grupo de pacientes.

Os dados da Tabela 2 mostram a incidência das variáveis que compõem o DEPI nos quatro grupos. Observou-se que a média dos indicadores de DEPI no grupo de pacientes tanto masculinos, quanto femininos foi $4,40 \mathrm{e}$ no grupo controle de 3,47 às mulheres e 3,33 aos homens. Comparando-se os resultados das variáveis contínuas pelos testes de KruskalWallis e post-hoc de Dunn para a comparação dos escores entre os quatro grupos, obteve-se $p$ $<0,001$, o que revelou uma diferença estatisticamente significativa entre eles, pois o grupo de pacientes possui um maior número de indicadores de DEPI quando comparado ao grupo-controle.

Desta maneira, é possível afirmar que os pacientes com Transtorno de Pânico que compuseram a amostra possuíam maior vivência depressiva do que os colaboradores do grupo de não pacientes. Verificou-se também que pacientes com pânico tendem a apresentar DEPI positivo quando avaliados pelo Rorschach, em virtude de uma possível vivência depressiva ou de uma propensão à alteração de humor com tendência à depressão.

Del Porto (2002) assinala que entre $40 \%$ e $80 \%$ dos casos de pânico estão associados a transtornos depressivos. O índice de DEPI positivo foi observado também por Exner e Erdberg (2005) em um estudo de um caso de pânico de um homem de 23 anos de idade, revelando que este sujeito encontrava-se vulnerável a situações de depressão e sofrimento afetivo.

A relação entre pânico e depressão também foi notada no estudo de Montiel, A. G. S. Capovilla, Berberian e F. C. Capovilla (2005), que observaram, baseados no Inventário de Beck, grande incidência de sintomas depressivos em pacientes com pânico. Os autores concluíram que existe uma forte relação entre os transtornos de ansiedade, sobretudo o pânico, com o desenvolvimento de sintomas depressivos. Explicaram que, quanto maior a vivência de ansiedade, maior seria a possibilidade do desenvolvimento de sintomas depressivos.

Em trabalho anterior, Caetano (1985) descreveu a grande ocorrência de sintomas depressivos em pacientes com transtornos fóbico-ansiosos, em especial, o transtorno de pânico. Entretanto, relatou que a depressão desenvolvida por pacientes com pânico é produzida quando o quadro é crônico e limitante. Resultados semelhantes foram 
observados por Andersen e Rosenberg (1990) que perceberam grande presença de sintomas depressivos em pacientes com pânico.

Diferentes estudos clínicos, desenvolvidos com diversos tipos de delineamento e levantamento de dados, também revelaram forte associação entre o pânico e quadros ou vivências depressivas (Blaya et al., 2006; Castro et al., 2009; Freire et al., 2007; Mathias, Mezzasalma, \& Nardi, 2011).
Pelo fato dos dados terem identificado forte incidência de quadros depressivos em comorbidade com Transtorno de Pânico, optouse por apresentar os indicadores do Índice de Depressão para que fosse possível a identificação das variáveis que compõem o referido índice. $\mathrm{O}$ detalhamento proporcionado pela análise dos diferentes itens oferece maior visão dos fatores que os pacientes apresentaram para caracterizar o quadro depressivo.

Tabela 3 - Comparação dos indicadores de DEPI de pacientes e não pacientes pelo Teste Exato de Fisher e post-doc de Dunn

\begin{tabular}{|c|c|c|c|c|c|c|}
\hline \multirow[t]{2}{*}{ Indicador } & \multicolumn{2}{|c|}{ Pacientes } & \multicolumn{2}{|c|}{ Não Pacientes } & \multirow[t]{2}{*}{ Estatística } & \multirow[t]{2}{*}{$p$} \\
\hline & $\%$ & $\mathrm{~F}$ & $\%$ & $\mathrm{~F}$ & & \\
\hline$F V+V F+V>0$ & 6,67 & 2 & 6,67 & 2 & Fisher & 1,000 \\
\hline $\mathrm{FD}>2$ & - & - & 3,33 & 1 & Fisher & 1,000 \\
\hline Col-Shad Blends $>0$ & 70,00 & 21 & 33,33 & 10 & $X^{2}=8,08$ & 0,005 \\
\hline$S>2$ & 6,67 & 2 & - & - & Fisher & 0,492 \\
\hline $\begin{array}{l}3 r+(2) / R>0.44 \text { e } \\
F r+r F=0\end{array}$ & 23,33 & 7 & 20,00 & 6 & $X^{2}=0,10$ & 0,754 \\
\hline $3 r+(2) / R<0.33$ & 63,33 & 19 & 43,33 & 13 & $X^{2}=2,41$ & 0,121 \\
\hline Afr $<0.46$ & 73,33 & 22 & 60,00 & 18 & $X^{2}=1,20$ & 0,273 \\
\hline Blends $<4$ & 43,33 & 13 & 66,67 & 20 & $X^{2}=3,30$ & 0,069 \\
\hline SumShading $>$ FM + m & 86,67 & 26 & 50,00 & 15 & $X^{2}=9,32$ & 0,002 \\
\hline SumC' $>2$ & 40,00 & 12 & 10,00 & 3 & $X^{2}=7,20$ & 0,007 \\
\hline$M O R>2$ & 3,33 & 1 & 3,33 & 1 & Fisher & 1,000 \\
\hline $2 A B+A r t+A y>3$ & 6,67 & 2 & & - & Fisher & 0,492 \\
\hline $\mathrm{COP}<2$ & 96,67 & 29 & 93,33 & 28 & Fisher & 1,000 \\
\hline Isolate/R > 0.24 & 46,67 & 14 & 33,33 & 10 & $X^{2}=1,11$ & 0,292 \\
\hline
\end{tabular}

Na Tabela 3, estão expostas as comparações da incidência dos indicadores de DEPI nos grupos de pacientes e não pacientes, onde é possível observar diferença estatisticamente significativa em três dados: Incidência de determinantes mistos de cor e sombreado (Col-Shad Blends > 0) que compõe um indicador; somatória de sombreado maior que movimentos animais e inanimados (SumShading > FM $+\mathrm{m}$ ) e somatória de determinantes de cor acromática maior que dois (SumC' > 2), esses últimos, juntos, compõem outro indicador.

Conforme assinala Castro (2008), os indicadores de depressão observados nos pacientes com pânico que participaram da presente pesquisa foram identificados por três condições em especial:

1 - Incidência de determinantes mistos de cor e sombreado

Este dado revela que os pacientes com pânico demonstram vivências afetivas confusas e dolorosas, equivale a dizer que ao experimentar qualquer tipo de afeto, este vem carregado de ambivalência e sofrimento.

Em um caso avaliado por Exner e Erdberg (2005), houve a incidência de um Col-Shad Blend, que já é considerado como um sinal de perturbação afetiva. As demais pesquisas envolvendo a avaliação de pacientes com pânico também indicaram, de alguma forma, que os sujeitos passam por vivências de sofrimento emocional que acarretam sensação de ambivalência e pouca organização de componentes afetivos, podendo levá-los a uma desorganização interna (Andersen \& Rosenberg, 1990; Caetano, 1985; Cohen \& Ruiter, 1992; MacFadden, 1994; Montiel et al., 2005; Morais \& Souza, 1996; VillemorAmaral, Farah, \& Primi, 2004).

2 - Predomínio de respostas de sombreado sobre movimentos

Esse aspecto pode ser observado, de forma empírica, nas pesquisas com pacientes com pânico, em que são sempre frequentes as indicações de sofrimento e dor psicológica 
vividas pelos sujeitos. Independentemente da estratégia metodológica que tenha sido adotada, os componentes de sofrimento psicológico são sempre presentes e representam um indicador característico dos quadros de pacientes com transtorno de pânico (Andersen \& Rosenberg, 1990; Caetano, 1985; Cohen \& Ruiter, 1992; MacFadden, 1994; Montiel et al., 2005; Morais \& Souza, 1996; Villemor-Amaral et al., 2004). 3 - Incidência de respostas de cor acromática

$\mathrm{O}$ dado indica que os pacientes não conseguem expressar seus conteúdos afetivos de forma clara e coerente, levando-os a certa constrição afetiva, reforçando as indicações de pesquisas feitas anteriormente.

\section{Índice de Transtornos de Percepção e Pensamento}

$\mathrm{O}$ Índice de Transtornos de Percepção e Pensamento - PTI é composto de seis elementos que se integram na avaliação de comprometimento na percepção ou no pensamento e mostra-se imperativo para a análise da ideação. Segundo normas brasileiras, quando há incidência de três ou mais indicadores, pode revelar a presença acentuada de algum tipo de patologia relacionada à ideação ou à mediação.

Tabela 4 - Incidência de indicadores de PTI nos quatro grupos pelo teste de Kruskal-Wallis

\begin{tabular}{lcccccc}
\hline Indic. & Média & D.P. & Min & Máx & Mediana & Sig \\
\hline PF & 0,80 & 1,26 & 0,00 & 3,00 & 0,00 & \\
PM & 1,47 & 1,13 & 0,00 & 3,00 & 2.00 & \multirow{2}{*}{$0=0,002$} \\
CF & 0,53 & 1,06 & 0,00 & 3,00 & 0,00 & \\
CM & 0,07 & 0,26 & 0,00 & 1,00 & 0,00 & \\
\hline
\end{tabular}

Os dados da Tabela 4 mostram a incidência das variáveis que compõem o PTI nos quatro grupos. Tem-se que a média dos indicadores de PTI no grupo de pacientes foi 0,80 para as mulheres e 1,47 para os homens com pânico, no grupo de não pacientes observa-se média de 0,53 às mulheres e 0,07 aos homens. Comparando-se os resultados das variáveis contínuas pelos testes de KruskalWallis e post-hoc de Dunn para a comparação dos escores entre os quatro grupos, obteve-se $p$ $=0,002, \quad$ o que revelou uma diferença estatisticamente significativa entre eles, pois os pacientes do sexo masculino com pânico possuem maior número de indicadores de PTI quando comparado ao grupo controle.

Desta maneira, é possível afirmar que os homens com Transtorno de Pânico que compuseram a amostra, por meio da avaliação do Rorschach, possuíam graves problemas na articulação perceptiva e de pensamento, podendo ser um sinal de vivência psicopatológicas na esfera da ideação e/ou da percepção.

Pesquisas normativas brasileiras apontam média para o PTI em 0,69 com desvio padrão em 1,12 (Nascimento, 2010). Dessa maneira, detecta-se que os valores para os pacientes do sexo masculino encontram-se acima do que foi apresentado pela população do país.

Villemor-Amaral, Franco e Farah (2008) observaram diminuição da qualidade e comprometimento do pensamento em pacientes com pânico, por meio da análise fenômenoestrutural das respostas do Método de Rorschach. Além delas, pesquisas anteriores também assinalaram algum tipo de prejuízo de pensamento nos quadros de pânico que levam os pacientes a algum tipo de comprometimento da ideação, com prejuízo às funções cognitivas e de contato com a realidade nesses indivíduos (Figueira et al., 1992; Mello, 1988).

Apesar do exposto, dados do estudo de Exner e Erdberg (2005) não indicaram de PTI no estudo de caso de paciente com pânico, cujo valor do índice foi zero.

\section{Índice de Déficit Relacional}

O Índice de Déficit Relacional - CDI é um escore obtido com base na observação de 11 variáveis que se agrupam em cinco elementos de análise com presença de, pelo menos, quatro desses elementos. Avalia a existência de dificuldades para enfrentar eficientemente as demandas comuns do meio social onde o indivíduo insere-se, revelando certa falta de aptidão ou dificuldade para os elementos 
relacionais, levando-os a problemas na interação com os demais sujeitos que 0 rodeiam. Apresentam características de distância dos demais, pouca sensibilidade às necessidades dos outros, levando-os a fracassos nos contatos interpessoais.
Quando os resultados do CDI dos quatro grupos foram comparados, nos dados das Tabelas 5 e 6, não houve diferenças estatisticamente significativas entre os dados de pacientes e não pacientes.

Tabela 5 - Presença de CDI nos quatro grupos pelo Teste Exato de Fisher

\begin{tabular}{|c|c|c|c|c|c|}
\hline \multirow[b]{2}{*}{ CDI } & \multicolumn{2}{|c|}{ Sim } & \multicolumn{2}{|c|}{ Não } & \multirow[t]{2}{*}{ Sig } \\
\hline & $\mathrm{N}$ & $\%$ & $\mathrm{~N}$ & $\%$ & \\
\hline PF & 11 & $\begin{array}{l}73,33 \\
\end{array}$ & 4 & 26,67 & \\
\hline PM & 11 & 73,33 & 4 & 26,67 & $p=0,891$ \\
\hline CF & 11 & 73,33 & 4 & 26,67 & \\
\hline CM & 9 & 60,00 & 6 & 40,00 & \\
\hline
\end{tabular}

Tabela 6 - Incidência de indicadores de CDI nos quatro grupos pelo teste de Kruskal-Wallis

\begin{tabular}{ccccccc}
\hline Indic. & Média & D.P. & Min & Máx & Mediana & Sig \\
\hline PF & 3,73 & 1,10 & 1,00 & 5,00 & 4,00 & \\
PM & 3,73 & 0,88 & 2,00 & 5,00 & 4,00 & $p=0,979$ \\
CF & 3,73 & 0,88 & 2,00 & 5,00 & 4,00 & \\
CM & 3,67 & 0,98 & 2,00 & 5,00 & 4,00 & \\
\hline
\end{tabular}

Os dados expostos na Tabela 5 demonstram que $73,33 \%(\mathrm{~N}=11)$ dos pacientes com pânico, bem como os não pacientes do sexo feminino indicaram CDI positivo, $60 \%$ $(\mathrm{N}=9)$ do grupo controle masculino também apresentaram a mesma indicação. Quando os dados das variáveis categóricas do CDI foram comparados pelo teste Exato de Fischer, obteve-se a diferença $\mathrm{p}=0,891$, revelando que não houve diferenças estatisticamente significativas entre os grupos.

As médias de variáveis positivas para CDI nos quatro grupos foram muito semelhantes. De acordo com a Tabela 6, encontraram-se 3,73 para os pacientes e controle feminino e 3,67 para controle masculino. Depois dos testes Kruskal-Wallis e post-hoc de Dunn para a análise das variáveis contínuas do CDI, obtevese um escore $\mathrm{p}=0,979$, que indica a ausência de diferenças significativas.

Nesses dados, a maioria dos indivíduos que compôs a amostra, indicou algum tipo de dificuldade de interação com outras pessoas. Como diferenças não foram observadas, esse aspecto não foi considerado como característica dos pacientes com pânico. Embora não tenha sido alvo da presente investigação, conjecturouse que outros fatores desenvolvimentais ou sociais podem ter interferido na dificuldade de interação presente nos componentes da amostra. Na avaliação de Exner e Erdberg (2005), embora o paciente com pânico não tenha indicado CDI positivo, apresentou três elementos que caracterizam tal índice.

\section{Constelação de Suicídio}

A constelação de suicídio - S-CON constitui-se em um índice composto a partir da verificação de 12 variáveis que se relacionam para sua avaliação, é considerado positivo quando o protocolo indicar oito ou mais condições assinaladas. Nesse caso, revela potencial autodestrutivo, podendo estar associado a risco de conduta ou ideação suicida.

Os dados da Tabela 7 apresentam os dados de S-CON demonstrados pelos sujeitos desta pesquisa. Observa-se que não existem diferenças entre os quatro grupos, pacientes com pânico feminino e masculino e controle feminino e masculino. 
Tabela 7 - Presença de S-Con nos quatro grupos pelo Teste Exato de Fisher

\begin{tabular}{|c|c|c|c|c|c|}
\hline \multirow[b]{2}{*}{ S-Con } & \multicolumn{2}{|c|}{ Sim } & \multicolumn{2}{|c|}{ Não } & \multirow[t]{2}{*}{ Sig } \\
\hline & $\mathrm{N}$ & $\%$ & $\mathrm{~N}$ & $\%$ & \\
\hline PF & 0 & 0,00 & 15 & 100,00 & \\
\hline PM & 1 & 6,67 & 14 & 93,33 & $p=1,000$ \\
\hline CF & 0 & 0,00 & 15 & 100,00 & \\
\hline CM & 1 & 6,67 & 14 & 93,33 & \\
\hline
\end{tabular}

Tabela 8 - Incidência de indicadores de S-Con nos quatro grupos pelo teste de Kruskal-Wallis

\begin{tabular}{ccccccc}
\hline Indic. & Média & D.P. & Min & Máx & Mediana & Sig \\
\hline PF & 5,00 & 1,07 & 3,00 & 7,00 & 5,00 & \\
PM & 5,20 & 1,47 & 3,00 & 8,00 & 5,00 & $p=0,093$ \\
CF & 4,00 & 1,41 & 1,00 & 7,00 & 4,00 & \\
CM & 4,47 & 1,68 & 2,00 & 8,00 & 4,00 & \\
\hline
\end{tabular}

O teste Exato de Fisher teve significância de $\mathrm{p}=1,000$. Houve um predomínio quase absoluto da pontuação negativa de S-CON, ou seja, quase a totalidade dos participantes do estudo não indicou a Constelação de Suicídio em seus protocolos do Rorschach. Apenas dois homens, um do grupo de pacientes e outro do grupo de não pacientes que pontuaram positivamente na referida constelação. No estudo de Exner e Erdberg (2005), o pacienta com pânico avaliado também não apresentou SCON positivo.

A comparação dos dados dos quatro grupos para os indicadores de S-CON, Tabela 9 , indicou $\mathrm{p}=0,093$. Após a aplicação dos testes de Kruskal-Wallis e post-hoc de Dunn, foi revelado que não existem diferenças estatisticamente significativas entre eles. As médias foram: para o grupo de pacientes femininos 5,00 e aos pacientes masculinos de 5,20 . Os dados do grupo de não pacientes demonstraram médias de 4,00 às mulheres e 4,47 aos homens.

\section{Índice de Hipervigilância}

O Índice de Hipervigilância - HVI é indicado pela presença de respostas de sombreado textura, associada a pelo menos quatro outras variáveis, identificadas em um conjunto de sete sinais. Quando presente, revela um estado de alerta contínuo e em alguns casos está associado a uma tendência paranoica, ou seja, sensação constante de ser observado e demasiadamente notado pelo ambiente e, em decorrência disso, uma atitude negativista diante das relações sociais.

Os dados expostos na Tabela 9, referentes à incidência de HVI nos quatro grupos, revela, após aplicação do teste Exato de Fisher, um valor de significância $\mathrm{p}=1,000$, revelando que não há diferença estatisticamente significativa entre os grupos. Apenas um paciente do sexo masculino apresentou o Índice de Hipervigilância. Exner e Erdberg (2005) levantaram HVI negativo em sua avaliação com paciente com pânico.

Tabela 9 - Presença de HVI nos quatro grupos pelo Teste Exato de Fisher

\begin{tabular}{llllll}
\hline & \multicolumn{2}{c}{ Sim } & \multicolumn{2}{c}{ Não } & Sig \\
\cline { 2 - 5 } HVI & $\mathrm{N}$ & $\%$ & $\mathrm{~N}$ & $\%$ & \\
\hline $\mathrm{PF}$ & 0 & 0,00 & 15 & 100,00 & \\
$\mathrm{PM}$ & 1 & 6,67 & 14 & 93,33 & $\mathrm{p}=1,000$ \\
$\mathrm{CF}$ & 0 & 0,00 & 15 & 100,00 & \\
$\mathrm{CM}$ & 0 & 0,00 & 15 & 100,00 & \\
\hline
\end{tabular}




\section{Índice de Estilo Obsessivo}

O Índice de Estilo Obsessivo - OBS é identificado a partir da combinação entre cinco variáveis associadas à referida questão. Quando positivo, significa que o indivíduo avaliado mostra tendência forte ao perfeccionismo e à meticulosidade, levando-o a ações mais minuciosas do que as demais pessoas.
Por fim, conforme é possível observar na Tabela 10, os dados dos pacientes e não pacientes foram absolutamente iguais, nenhum participante indicou presença positiva do Estilo Obsessivo. Em avaliação realizada em jovem com pânico o OBS também foi negativo (Exner \& Erdberg, 2005).

Tabela 10 - Presença OBS de nos quatro grupos pelo Teste Exato de Fisher

\begin{tabular}{llllll}
\hline & \multicolumn{3}{c}{ Sim } & \multicolumn{3}{c}{ Não } & Sig \\
\cline { 2 - 5 } OBS & $\mathrm{N}$ & $\%$ & $\mathrm{~N}$ & $\%$ & \\
\hline PF & 0 & 0,00 & 15 & 100,00 & \\
PM & 0 & 0,00 & 15 & 100,00 & $\mathrm{p}=1,000$ \\
CF & 0 & 0,00 & 15 & 100,00 & \\
CM & 0 & 0,00 & 15 & 100,00 & \\
\hline
\end{tabular}

\section{Considerações finais}

A vivência de um quadro psicopatológico gera profundas consequências nas vidas dos pacientes e seus familiares. Tal situação se intensifica quando o sofrimento mental é acompanhado de outros quadros associados, configurando-se circunstâncias de comorbidade.

O presente estudo buscou avaliar a incidência de comorbidades em uma amostra de pacientes com Transtorno de Pânico, observando-se que a maior ocorrência de quadros psicopatológicos associados ao pânico foi o de depressão.

Na mostra investigada, a maior parte dos pacientes com pânico indicou depressão ou sinais de depressão como quadro associado. Importante salientar que, na constituição da amostra, foram selecionados pacientes sem comorbidades diagnosticadas pela entrevista ou pelo tratamento psiquiátrico. Os indicadores depressivos surgiram apenas nos dados do Método de Rorschach e foram levantados por meio do material de interpretação do instrumento.

Observa-se alta incidência de comorbidade entre pânico e depressão na amostra investigada, corroborado pelos estudos teóricos e empíricos sobre o assunto. Esses dados revelam a necessidade de se atentar para sinais depressivos em pacientes com pânico, mesmo que esses não sejam identificados em entrevistas iniciais.
Outro fator de comorbidade foi o de alteração ou perturbação do pensamento, nesse caso apenas em homens com pânico, revelando certo comprometimento da articulação da ideação e/ou percepção desses pacientes.

Outros fatores identificados pelo Rorschach não mostraram incidência significativa e permitem afirmar que não existiram outros quadros em comorbidade com pânico.

Necessário salientar que os dados aqui expostos referem-se à amostra investigada e para generalizações mais amplas será necessária a ampliação do estudo. Além disso, necessário investigar outros possíveis quadros associados ao pânico que não foram investigados por não fazerem parte do grupo de sinais psicopatológicos avaliados pelo Rorschach.

\section{Referências}

Acklin, M. W. (1995). Integrative Rorschach Interpretation. Journal of Personality Assessment, 64 (2), 235-238.

Adrados, I., \& Figueiredo, N. (1995). Síndrome do Pânico. Boletim da Sociedade de Rorschach de São Paulo, 8(1), 42-49.

American Psychiatric Association (1995). DSM-IV - Manual diagnóstico e estatístico de transtornos mentais (4a ed.). (D. Batista, trad.). Porto Alegre: Artes Médicas. 
Andersen, R., \& Rosenberg, N. K. (1990). Rorschach-profile in panic disorder. Scandinavian Journal of Psychology, 31, 99-109.

Arbona, C. B., \& Arnal, R. B. (1995). Transtorno por pánico. In G. Buela-Casal, V. E. Caballo, \& J. A. Carrobles (Dirs.), Manual de psicopatología y transtornos psiquiátricos (pp. 365-407). Madrid: Siglo Veintiuno de España.

Barlow, D. H., \& Cerny, J. A. (1988/1999). Tratamento psicológico do pânico (M. C. Monteiro, trad.). Porto Alegre: Artes Médicas.

Blaya, C. et al. (2006). Do defense mechanisms vary acoording to the psychiatric disorder? Revista Brasileira de Psiquiatria, 28(3), 179-83.

Brasil (1996). Ministério da Saúde. Conselho Nacional de Saúde. Fundação Nacional de Saúde. Diretrizes e Normas Regulamentadoras de Pesquisas Envolvendo Seres Humanos. Brasília: Ministério da Saúde.

Caetano, D. (1985). Relação entre ataque de pânico e depressão. Jornal Brasileiro de Psiquiatria, 31(1), 17-24.

Campo, V. (1995). Estudios clínicos con el Rorschach en niños, adolescentes y adultos. Barcelona: Paidós.

Castro, M. et al. (2009). Comorbid anxiety and depression disorders in patients with chronic pain. Arquivos de Neuropsiquiatria, 67(4), 982-985.

Castro, P. F. (2008). Caracterização da personalidade de pacientes com Transtorno de Pânico por meio do Método de Rorschach: contribuições do sistema compreensivo. São Paulo, Tese de Doutorado, Instituto de Psicologia da Universidade de São Paulo, São Paulo.

Chabert, C. (1987/1993). A Psicopatologia no Exame de Rorschach (N. Silva Júnior, trad.). São Paulo: Casa do Psicólogo.

Cheniaux, E. (2011). Manual de Psicopatologia (4 ed.). Rio de Janeiro: Guanabara Koogan.
Cohen, L., \& Ruiter, C. (1992). Personality in Panic Disorder with Agoraphobia: a Rorschach study. Journal of Personality Assessment, 59(2), 304-316.

Cohen, L., \& Ruiter, C. (1993). Persoonlijkheidskenmerken van patienten met agorafobie: een studie met het Comprehensive System voor de Rorschach. Nederlands Tijdschrift voor de Psychologie en haar Grensgebieden, 48(1), 35-42.

Cunha, J. A. (1996). Avaliação psicológica. In E. D. Busnello, P. Prado-Lima \& J. G. V. Taborda, Rotinas em psiquiatria (pp. 5057). Porto Alegre: Artes Médicas.

Cunha J. A. (2000). Catálogo de técnicas úteis. In J. A. Cunha et al., Psicodiagnóstico - V (5a ed.; pp. 202-290). Porto Alegre: Artes Médicas.

Dalgalarrondo, P. (2008). Psicopatologia $e$ semiologia dos Transtornos Mentais (2 ed.). Porto Alegre: Artmed.

Del Porto, J. A. (2002). Diagnóstico e tratamento da Síndrome do Pânico. In J. J. Mari et al., Psiquiatria (pp. 19-29). São Paulo: Manole.

Del-Ben, C. M. (2004). Transtorno do pânico. In L. A. B. Hetem, \& F. G. Graeff (Orgs.), Transtorno de ansiedade (pp. 207-230). São Paulo: Editora Atheneu.

Exner, J. E. (1993/1994). El Rorschach: un sistema comprehensivo (3a ed.). (M. E. Ramírez, trad.). Madrid: Psimática.

Exner, J. E. (1995/1999). Manual de classificação do Rorschach para o sistema compreensivo. (A. C. P. Silva Neto, trad.). São Paulo: Casa do Psicólogo.

Exner, J. E. (Ed.) (1995). Issues and Methods in Rorschach Research. New Jersey: Lawrence Erbaum Associates Publishers.

Exner, J. E., \& Erdberg, P. (2005). The Rorschach: a comprehensive system advanced interpretation. (3rd ed.). New Jersey: John Wiley \& Sons, Inc.

Exner, J. E., \& Sendín, C. (1998/1999). Manual de interpretação do Rorschach para o sistema compreensivo. (L.Y. Massuh, trad.). São Paulo: Casa do Psicólogo. 
Farah, F. H. Z., \& Villemor-Amaral, A. E. (2008). O Transtorno do Pânico e o Rorschach no sistema compreensivo. Boletim de Psicologia, 58(128), 103-119.

Figueira, I. et al. (1992). Fobia social e transtorno do pânico em pacientes psicóticos. Jornal Brasileiro de Psiquiatria, 41(6), 259-264.

Frank, G. (1997). Research assessment of the clinical utility of the Rorschach. Psychological Reports, 81, 1255-1258.

Freire, R. C. et al. (2007). Personality traits spectrum in panic disorder and major depression. Revista Brasileira de Psiquiatria, 29(1), 31-34.

Gentil, V. (1996). Transtorno do pânico. In E. D. Busnello, P. Prado-Lima \& J. G. V. Taborda, Rotinas em psiquiatria (pp. 171177). Porto Alegre: Artes Médicas.

Gentil, V. (1997a). Ansiedade e transtornos ansiosos. In V. Gentil, F. Lotufo-Neto, \& M. A. Bernik (Orgs.), Pânico, fobias $e$ obsessões: a experiência do Projeto AMBAN (3a ed.; pp. 29-36). São Paulo: EDUSP.

Gentil, V. (1997b). Transtorno de pânico. In V. Gentil, F. Lotufo-Neto, \& M. A. Bernik (Orgs.), Pânico, fobias e obsessões: a experiência do Projeto AMBAN (3a ed.; pp. 101-111). São Paulo: EDUSP.

Gentil, V., Ito, L. M., \& Roso, M. C. (1987). Problemas da determinação de um perfil de personalidade em pacientes com síndrome do pânico. Revista Associação Brasileira de Psiquiatria - Asociacion Psiquiatrica de la America Latina, 9(4), 140-144.

Goldberg, D. P. (1972/1996). Questionário de saúde geral de Goldberg: Manual técnico (L. Pasquali, V. V. Gouveia, W. B. Andriola, F. J. Miranda \& A. L. M. Ramos, trad. e adapt.). São Paulo: Casa do Psicólogo.

Hernández, M. M. (1993). Metodología aplicada a la investigación en psicología. Madrid: Editorial Complutense.

Hübner-D’Oliveira, M. M. (1984). Ciência e pesquisa em psicologia: uma introdução. São Paulo: E.P.U.
Ito, L. M., \& Ramos, R. T. (1998). Escalas de avaliação clínica: transtorno de pânico. Revista de Psiquiatria Clínica, 25(6 Edição Especial), 294-302.

Kaplan, H. I., \& Sadock, B. J. (1991-1993). Compêndio de psiquiatria: Ciências comportamentais e psiquiatria clínica (6a ed.; D. Batista, trad.). Porto Alegre: Artes Médicas.

Levin, J. (1987). Estatística aplicada a Ciências Humanas (2a ed.; S. F. Costa, Trad). São Paulo: Harbra.

López, A. B. (1995). Agorafobia I: Naturaleza, etiologia y evaluación. Barcelona: Ediciones Paidós Ibérica.

López, A. B. (2000). Agorafobia y Ataques de Pánico. Madrid: Ediciones Pirámide.

MacFadden, M. A. (1994). Avaliação psicodinâmica de mulheres com transtorno de pânico. Boletim de Psicologia, 44(100/101), 49-53.

Mathias, K. V., Mezzasalma, M. A., \& Nardi, A.,E. (2011). Prevalência de transtorno de pânico em pacientes com zumbidos. Revista de Psiquiatria Clínica, 38(4), 139-42.

Mello, M. F (1988). Considerações sobre o distúrbio de pânico. Boletim do Centro de Estudos em Pesquisa Psiquiátrica, 6(1/2) 47-50.

Montiel, J. M., Capovilla, A. G. S., Berberian , A. A., \& Capovilla, F. C. (2005). Incidência de sintomas depressivos em pacientes com transtorno de pânico. Psic, 6(2), 33-42.

Morais, M. A. V., \& Souza, A. M. (1996). Avaliação da personalidade de pacientes do distúrbio do pânico, com o uso de técnicas projetivas (pp.101-107). In Anais, 1. Encontro da Sociedade Brasileira de Rorschach e outros métodos projetivos, 1996, Ribeirão Preto, SBRo.

Nascimento, R. S. G. F. (2010). Sistema compreensivo do Rorschach: teoria, pesquisa e normas para a população brasileira. São Paulo: Casa do Psicólogo. 
Organização Mundial de Saúde (Coord) (1992/1993). Classificação de transtornos mentais e de comportamento da CID-10: Descrições clínicas e diretrizes diagnósticas. (D. Caetano, trad.). Porto Alegre: Artes Médicas.

Pereira, J. C. R. (1999). Análise de dados qualitativos - Estratégias metodológicas para as Ciências da Saúde, Humanas e Sociais. São Paulo: EDUSP.

Ramos, R. T. (1995). Psicopatologia do Transtorno do Pânico: Efeito da terapêutica e delimitação diagnóstica. São Paulo, Tese de Doutorado, Faculdade de Medicina, Universidade de São Paulo, São Paulo.

Santos, M. A. (1996). Aplicações da Prova de Rorschach no campo da psicopatologia (pp. 257-281). In Anais, 1. Encontro da Sociedade Brasileira de Rorschach e outros métodos projetivos, 1996, Ribeirão Preto, SBRo.

Sarvasi, M. M. O. C. (1999). A Técnica de Rorschach na investigação das relações objetais de pacientes com Transtorno de Pânico. São Bernardo do Campo, Dissertação de Mestrado, Universidade Metodista de São Paulo, São Bernardo do Campo.

Severino, A. J. (2001). Metodologia do trabalho científico (21a ed. rev. ampl.). São Paulo: Cortez Editora.

Siegel, S. (1975). Estatística não-paramétrica para as Ciências do Comportamento. São Paulo: McGraw-Hill.
Troiano, M. (2001). Guarire dagli attacchi di panico. Roma: Riuniti.

Vaz, C. E. (1997). O Rorschach: Teoria e desempenho (3a ed.). São Paulo: Manole.

Vieira, W. C. (1997). A síndrome do pânico. Perfil, 10, 17-23.

Villemor-Amaral, A. E., Farah, F. H. Z., \& Primi, R. (2004). O teste das pirâmides coloridas e o transtorno de pânico. Psicologia em Estudo, 9(2), 301-307.

Villemor-Amaral, A. E., Franco, R. R. C., \& Farah, F. H. Z. (2008). A psicopatologia fenômeno-estrutural e o Rorschach no transtorno de pânico. Estudos de Psicologia, 25(1), 141-150.

Weiner, I.B. (1986). Conceptual end empirical perspectives on the Rorschach assessment of psychopathology. Journal of Personality Assessment, 50 (3), 472-479.

Weiner, I.B. (1988-2000). Princípios de Interpretação do Rorschach (M. C. V. Silva, Trad.). São Paulo: Casa do Psicólogo.

Yazigi, L. (2010). Fundamentação teórica do Método de Rorschach. In S. R. Pasian (Org.), Avanços do Rorschach no Brasil (pp. 7-29). São Paulo: Casa do Psicólogo.

Recebido em 21 de Março de 2012 Texto reformulado em 24 de Outubro de 2012 Aceite em 25 de Outubro de 2012 Publicado em 31 de Dezembro de 2012 